PROCEEDINGS OF THE

AMERICAN MATHEMATICAL SOCIETY

Volume 132, Number 4, Pages 1125-1131

S 0002-9939(03)07243-5

Article electronically published on November 4, 2003

\title{
THE SPLITTING PROBLEM FOR SUBSPACES OF TENSOR PRODUCTS OF OPERATOR ALGEBRAS
}

\author{
JON KRAUS
}

(Communicated by David R. Larson)

\begin{abstract}
The main result of this paper is that if $N$ is a von Neumann algebra that is a factor and has the weak* operator approximation property (the weak* OAP), and if $R$ is a von Neumann algebra, then every $\sigma$-weakly closed subspace of $N \bar{\otimes} R$ that is an $N \bar{\otimes} \mathbb{C} 1_{R}$-bimodule (under multiplication) splits, in the sense that there is a $\sigma$-weakly closed subspace $T$ of $R$ such that $S=N \bar{\otimes} T$. Note that if $S$ is a von Neumann subalgebra of $N \bar{\otimes} R$, then $S$ is an $N \bar{\otimes} \mathbb{C} 1_{R}$-bimodule if and only if $N \bar{\otimes} \mathbb{C} 1_{R} \subset S$. So this result is a generalization (in the case where $N$ has the weak* OAP) of the result of Ge and Kadison that if $N$ is a factor, then every von Neumann subalgebra $M$ of $N \bar{\otimes} R$ that contains $N \bar{\otimes} \mathbb{C} 1_{R}$ splits. We also obtain other results concerning the splitting of $\sigma$-weakly closed subspaces of tensor products of von Neumann algebras and the splitting of normed closed subspaces of $\mathrm{C}^{*}$-algebras that generalize results previously obtained for von Neumann subalgebras and $\mathrm{C}^{*}$-subalgebras.
\end{abstract}

In this article we are concerned with the following question: if $N$ and $R$ are von Neumann algebras, and if $S$ is a $\sigma$-weakly closed $N \bar{\otimes} \mathbb{C} 1_{R}$-bimodule of $N \bar{\otimes} R$, when do we have

$$
S=N \bar{\otimes} T
$$

for some $\sigma$-weakly closed subspace $T$ of $R$ ? If (1) holds for some $T$, then we say that $S$ splits. Note that if a $\sigma$-weakly closed subspace $S$ of $N \bar{\otimes} R$ splits, then it is an $N \bar{\otimes} \mathbb{C} 1_{R}$-bimodule. So this requirement is necessary for splitting. This problem has been previously studied in the case when $S=M$ is a von Neuman subalgebra of $N \bar{\otimes} R$ containing $N \bar{\otimes} \mathbb{C} 1_{R}$ (which is obviously equivalent to $M$ being an $N \bar{\otimes} \mathbb{C} 1_{R^{-}}$ bimodule). Ge and Kadison showed in 4 that if $N$ is a factor, then every von Neumann algebra $M$ satisfying $N \bar{\otimes} \mathbb{C} 1_{R} \subset M \subset N \bar{\otimes} R$ splits. In [10] Strătilă and Zsidó extended this result by showing that if $N$ is a von Neumann algebra with center $Z(N)$, and if $H$ is a Hilbert space, then a von Neumann algebra $M$ such that $N \bar{\otimes} \mathbb{C} 1_{B(H)} \subset M \subset N \bar{\otimes} B(H)$ is of the form $N \bar{\otimes} P$ for some von Neumann subalgebra $P$ of $B(H)$ if and only if $M \cap(Z(N) \bar{\otimes} B(H))=Z(N) \bar{\otimes} P$. By modifying the methods of [10], we are able to extend their result (and so the result of Ge and Kadison) to the case where $S$ is a $\sigma$-weakly closed subspace of $N \bar{\otimes} R$ that is an $N \bar{\otimes} \mathbb{C} 1_{R}$-bimodule. However, we have to add a condition to $N$, namely that $N$ satisfies Property $S_{\sigma}$ (introduced by the author in [6]) or, equivalently, the weak* operator approximation property. We also observe that the proof of the main result

Received by the editors June 14, 2002 and, in revised form, December 13, 2002.

2000 Mathematics Subject Classification. Primary 46L10. 
in [16] (and Theorem 3.3 in [15]) can be easily modified to give a splitting result for certain norm closed subspaces of the spatial (= minimal) tensor product of $\mathrm{C}^{*}$ algebras. We would like to thank the referee for helpful comments and suggestions.

Our first result is an extension of Theorem 3.5 in [10. Since the proof of Theorem 1 is essentially the same as the proof of Theorem 3.5 in [10, it is omitted. (The only difference between Theorem 1 below and Theorem 3.5 in 10 is that in 10 . it is shown that if $N \subset M \subset N \vee N_{0}$ is an intermediate von Neumann algebra, then $\Phi(M)=M \cap N_{0}=N^{\prime} \cap M$ rather than $\Phi(S)=S \cap N_{0}=N^{\prime} \cap S$ if $S$ is a $\sigma$-weakly closed subspace of $N \vee N_{0}$ that is an $N$-bimodule. The only place in the proof of the equality $\Phi(M)=M \cap N_{0}$ in [10] where the fact that $M$ is a von Neumann algebra is used is that $N \subset M$ implies that $M$ is an $N$-bimodule, while the proof in [10] of the equality $M \cap N_{0}=N^{\prime} \cap M$ is valid for any subset $M$ of $N \vee N_{0}$.)

Theorem 1. Let $N, N_{0} \subset B(H)$ be commuting von Neumann algebras with common center $Z$, and suppose $N_{0}$ is type $I$. Then

(1) $\left.\Phi \rightarrow \Phi\right|_{N}$ establishes a one-to-one correspondence between normal conditional expectations $\Phi: N \vee N_{0} \rightarrow N_{0}$ and normal conditional expectations from $N$ to $Z$;

(2) the normal conditional expectations $N \vee N_{0} \rightarrow N_{0}$ separate the points of $N \vee N_{0}$

(3) for every normal conditional expectation $\Phi: N \vee N_{0} \rightarrow N_{0}$ and every $\sigma$-weakly closed subspace $S$ of $N \vee N_{0}$ that is an $N$-bimodule, we have

$$
\Phi(S)=S \cap N_{0}=N^{\prime} \cap S .
$$

The proof of our main result (Theorem 2) uses Fubini products. So before proving the result we recall some of the basic definitions and results that we need. (See [6] and [7] for more details.) If $N$ and $R$ are von Neumann algebras, then for each $\phi \in N_{*}$, the right slice map $R_{\phi}: N \bar{\otimes} R \rightarrow R$ is defined by $R_{\phi}=\phi \otimes i d_{R}$. Note that $R_{\phi}$ is the unique $\sigma$-weakly continuous linear map from $N \bar{\otimes} R \rightarrow R$ satisfying

$$
R_{\phi}(a \otimes b)=\phi(a) b, \quad a \in N, b \in R .
$$

Similarly, if $\psi \in R_{*}$, the left slice map $L_{\psi}: N \bar{\otimes} R \rightarrow N$ is defined by $L_{\psi}=i d_{N} \otimes \psi$. If $S$ and $T$ are $\sigma$-weakly subspaces of $N$ and $R$ respectively, then the Fubini product of $S$ and $T$ is the $\sigma$-weakly closed subspace of $N \bar{\otimes} R$ defined by

$$
F(S, T)=\left\{x \in N \bar{\otimes} R: R_{\phi}(x) \in T \text { and } L_{\psi} \in S \text { for all } \phi \in N_{*} \text { and } \psi \in R_{*}\right\} .
$$

It is shown in [6] that $F(S, T)$ only depends on $S$ and $T$ and not on the containing von Neumann algebras. So if $N \subset B(H)$ and $R \subset B(K)$, we can replace $N$ by $B(H)$ and $R$ by $B(K)$ in the definition of $F(S, T)$. It is easy to see that we always have $S \bar{\otimes} T \subset F(S, T)$. A $\sigma$-weakly closed subspace $S$ of $B(H)$ is said to have Property $S_{\sigma}$ if $F(S, T)=S \bar{\otimes} T$ for all $\sigma$-weakly subspaces $T \subset B(K)$ for any Hilbert space $K$ (as shown in [6], it suffices to consider the case where $K$ is separable and infinite dimensional). If $1_{B(H)} \in S \subset B(H)$ and $1_{B(K)} \in T \subset B(K)$ are $\sigma$-weakly closed subspaces, then $(S \bar{\otimes} T)^{\prime}=F\left(S^{\prime}, T^{\prime}\right)$ (see [6]). In particular, if $N$ and $R$ are von Neumann algebras, then with $S=N^{\prime}$ and $T=R^{\prime}$, we get that

$$
F(N, R)=\left(N^{\prime} \bar{\otimes} R^{\prime}\right)^{\prime}=N^{\prime \prime} \bar{\otimes} R^{\prime \prime}=N \bar{\otimes} R
$$

by Tomita's Commutation Theorem, and, in fact, the above calculation shows that Tomita's Commutation Theorem is equivalent to the statement that $F(N, R)=$ 
$N \bar{\otimes} R$ whenever $N$ and $R$ are von Neumann algebras. However, not only are there subspaces $S$ and $T$ such that $F(S, T) \neq S \bar{\otimes} T$, but it is shown in [7] that for each of the types $I I_{1}, I I_{\infty}$ and $I I I_{\lambda}, 0 \leq \lambda \leq 1$, there is a separably acting factor $N$ of that type and a unital $\sigma$-weakly closed subalgebra $T$ of $B(K)$ (where $K$ is a separable infinite-dimensional Hilbert space) such that $N \bar{\otimes} T \neq F(N, T)$. (We cannot choose $N$ to be type I, because all type I von Neumann algebras have Property $S_{\sigma}[6]$ Theorem 1.9].) It is also shown in 7 that a subspace $S \subset B(H)$ has Property $S_{\sigma}$ if and only if it has the weak* OAP. (A subspace $S \subset B(H)$ has the weak* OAP if there is a net $\left\{\Phi_{i}\right\}$ of normal finite rank completely bounded maps from $S$ to $S$ such that for any Hilbert space $K$ and for any $x \in S \bar{\otimes} B(K)$, we have that $\left(\Phi_{i} \otimes i d_{B(K)}\right)(x) \rightarrow x \sigma$-weakly. See [3] for a detailed treatment of the weak* OAP and other approximation properties of operator spaces.)

Theorem 2. Let $N$ be a von Neumann algebra with center $Z(N)$, let $R$ be a von Neumann algebra, and suppose $S$ is a $\sigma$-weakly closed subspace of $N \bar{\otimes} R$ that is a $N \bar{\otimes} \mathbb{C} 1_{R}$-bimodule such that

$$
S \cap(Z(N) \bar{\otimes} R)=Z(N) \bar{\otimes} T
$$

for some $\sigma$-weakly closed subspace $T$ of $R$. Then

$$
N \bar{\otimes} T \subset S \subset F(N, T) .
$$

In particular, if $N$ has the weak* $O A P$, or if $S$ is a von Neumann algebra, then

$$
S=N \bar{\otimes} T \text {. }
$$

Proof. We can assume that $R \subset B(H)$ for some Hilbert space $H$. Then $S \subset$ $N \bar{\otimes} R \subset N \bar{\otimes} B(H)$, and $S$ is an $N \bar{\otimes} \mathbb{C} 1_{B(H)}$-bimodule. Moreover, we have that $S \cap$ $(Z(N) \bar{\otimes} B(H))=(S \cap(N \bar{\otimes} R)) \cap(Z(N) \bar{\otimes} B(H))=S \cap((N \bar{\otimes} R) \cap(Z(N) \bar{\otimes} B(H)))=$ $S \cap(Z(N) \bar{\otimes} R)=Z(N) \bar{\otimes} T$. Let $\phi \in N_{*}$ be a normal state, let $\psi=\left.\phi\right|_{Z(N)}$, and let $e$ be the support projection of $\psi$. Then there exists a positive normal $Z(N)$-module map $\Phi_{1}$ from $N$ into $Z(N)$ such that $\phi=\psi \circ \Phi_{1}$ and such that $\Phi_{1}(1)=e$ (see Theorem 1 in [5] or Proposition 1.4 in [9]). It follows from this result (applied to all normal states of $N$ ) and a Zorn's lemma argument that the normal conditional expectations from $N$ onto $Z(N)$ separate the points of $N$. Now let $\Phi_{2}$ be any normal conditional expectation from $N$ onto $Z(N)$, and define $\Phi$ on $N$ by $\Phi(x)=$ $\Phi_{1}(x)+(1-e) \Phi_{2}(x)$. Then $\Phi$ is a normal conditional expectation from $N$ onto $Z(N)$ such that $\phi=\psi \circ \Phi$. Since $\Phi \otimes i d_{B(H)}$ is a normal conditional expectation from $\left(N \bar{\otimes} \mathbb{C} 1_{B(H)}\right) \vee(Z(N) \bar{\otimes} B(H))=N \bar{\otimes} B(H)$ to $Z(N) \bar{\otimes} B(H)$, it follows from Theorem 1 that

$$
\left(\Phi \otimes i d_{B(H)}\right)(x) \in S \cap(Z(N) \bar{\otimes} B(H))=Z(N) \bar{\otimes} T \text { for all } x \in S,
$$

and so $R_{\psi}\left(\left(\Phi \otimes i d_{B(H)}\right)(x)\right) \in T$. But

(2) $R_{\psi}\left(\left(\Phi \otimes i d_{B(H)}\right)(x)\right)=\left(\psi \otimes i d_{B(H)}\right) \circ\left(\Phi \otimes i d_{B(H)}\right)(x)=\phi \otimes i d_{B(H)}(x)=R_{\phi}(x)$,

and so $R_{\phi}(x) \in T$ whenever $x \in S$ and $\phi$ is a normal state of $N$. Since the map $\phi \rightarrow R_{\phi}$ is linear, and since every $\phi \in N_{*}$ is a linear combination of normal states, we also have that $R_{\phi}(x) \in T$ for all $\phi \in N_{*}$. Moreover, since $S \subset N \bar{\otimes} R, L_{\psi}(x) \in N$ for all $\psi \in R_{*}$. Hence $S \subset F(N, T)$. By assumption, $Z(N) \bar{\otimes} T \subset S$ and $S$ is an $N \bar{\otimes} \mathbb{C} 1_{R}$-bimodule; so $\left(N \bar{\otimes} \mathbb{C} 1_{R}\right)(Z(N) \bar{\otimes} T)=N \bar{\otimes} T \subset S$. Hence $N \bar{\otimes} T \subset S \subset$ $F(N, T)$. As noted above, if $N$ has the weak* OAP, then $N$ has Property $S_{\sigma}$; so in this case we always have $N \bar{\otimes} T=F(N, T)$. Finally, if $S$ is a von Neumann algebra, 
then $S \cap(Z(N) \bar{\otimes} R)=Z(N) \bar{\otimes} T$ is also a von Neumann algebra, and so $T$ is a von Neumann algebra. Hence we again have $F(N, T)=N \bar{\otimes} T$.

If $N$ is a factor, then

$$
S \cap(Z(N) \bar{\otimes} R)=S \cap\left(\mathbb{C} 1_{N} \bar{\otimes} R\right) \subset \mathbb{C} 1_{N} \bar{\otimes} R=\left\{1_{N} \otimes b: b \in R\right\} .
$$

So we always have $S \cap(Z(N) \bar{\otimes} R)=\mathbb{C} 1_{N} \bar{\otimes} T=Z(N) \bar{\otimes} T$, where $T$ is a $\sigma$-weakly closed subspace of $R$. (In fact, $T=\left\{R_{\phi}(x): x \in S \cap(Z(N) \bar{\otimes} R)\right.$ and $\left.\phi \in N_{*}\right\}$.) Thus we get the following extension of the result of Ge and Kadison.

Theorem 3. Let $N$ be a factor, let $R$ be a von Neumann algebra, and suppose $S$ is a $\sigma$-weakly closed subspace of $N \bar{\otimes} R$ that is an $N \bar{\otimes} \mathbb{C} 1_{R}$-bimodule. Then there is a $\sigma$-weakly closed subspace $T$ of $R$ such that

$$
N \bar{\otimes} T \subset S \subset F(N, T) .
$$

In particular, if $N$ has the weak* $O A P$, or if $S$ is a von Neumann algebra, then

$$
S=N \bar{\otimes} T .
$$

Our next result shows that the requirement in Theorem 3 that $N$ have the weak* OAP is necessary for all $\sigma$-weakly closed $N \bar{\otimes} \mathbb{C} 1_{B(H)}$-bimodules of $N \bar{\otimes} B(H)$ to split.

Theorem 4. Suppose $N$ is a factor without the weak* $O A P$, and that $H$ is a separable infinite-dimensional Hilbert space. Then there is a unital (but not selfadjoint) $\sigma$-weakly closed subalgebra $S$ of $N \bar{\otimes} B(H)$ that is an $N \bar{\otimes} \mathbb{C} 1_{B(H)}$-bimodule, but which does not split.

Proof. Since $N$ is a von Neumann algebra without the weak* OAP, and so without Property $S_{\sigma}$, there is an abelian subalgebra $A$ of $B(H)$ that is reflexive (and so $\sigma$-weakly closed and unital) such that $F(N, A) \neq N \bar{\otimes} A$. (See Remark 1.1 in [7], noting that von Neumann algebras are always reflexive.) Let $S=F(N, A)$. Then $S$ is a $\sigma$-weakly closed unital subalgebra of $N \bar{\otimes} B(H)$. (See Remark 3.16 in [7.) For $\phi \in N_{*}$ and for $a \in N$, define the normal linear functionals $a \phi$ and $\phi a$ on $N$ by

$$
a \phi(b)=\phi(a b) \text { and } \phi a(b)=\phi(b a) \text { for all } b \in N \text {. }
$$

Then for all $\phi \in N_{*}, a \in N$, and $x \in N \bar{\otimes} B(H)$, we have

$$
R_{\phi}\left(\left(a \otimes 1_{B(H)}\right) x\right)=R_{a \phi}(x) \text { and } R_{\phi}\left(x\left(a \otimes 1_{B(H)}\right)\right)=R_{\phi a}(x),
$$

as can be easily seen (first check the equalities in (3) for $x$ of the form $x=b \otimes c$ ). It follows immediately from (3) that if $x \in F(N, A)$, then $\left(a \otimes 1_{B(H)}\right) x$ and $x\left(a \otimes 1_{B(H)}\right)$ are also in $F(N, A)$ for all $a \in N$; so $S$ is an $N \bar{\otimes} \mathbb{C} 1_{B(H)}$-bimodule. Finally, note that if $S=N \bar{\otimes} T$ for some $\sigma$-weakly closed subspace $T$ of $B(H)$, then $T=A$, which contradicts $F(N, A) \neq N \bar{\otimes} A$. Hence $S$ does not split (and so is not selfadjoint).

Combining Theorems 3 and 4, we get the following result.

Theorem 5. A factor $N$ has Property $S_{\sigma}$ if and only if for every von Neumann algebra $R$ (or just $R=B(H)$ ), every $\sigma$-weakly closed $N \bar{\otimes} \mathbb{C} 1_{R}$-bimodule of $N \bar{\otimes} R$ splits.

The von Neumann subalgebra version of Theorem 3 in 4 is used to show the following result ([4, Theorem $\mathrm{H}]$ ): if $N$ and $R$ are factors, if $N$ is injective, and if $P$ is a maximal injective von Neumann subalgebra of $R$, then $N \bar{\otimes} P$ is a maximal 
injective von Neumann subalgebra of $N \bar{\otimes} R$. Injectivity makes sense for norm closed subspaces of $B(H)$, when these are viewed as operator spaces. By definition, an operator space $V$ is injective if for any operator space $W_{0} \subset W$, every complete contraction from $W_{0}$ to $V$ has a completely contractive extension from $W$ to $V$. Since $B(H)$ is injective, it is not hard to show that an operator space $V \subset B(H)$ is injective if and only if there is a completely contractive projection from $B(H)$ onto $V$. (See [3 Proposition 4.1.6].) Using ideas from the proof of Theorem H, we obtain the next result.

Theorem 6. Let $N$ be an injective factor, and let $R$ be a von Neumann algebra. Then

(1) if $T$ is a maximal $\sigma$-weakly closed injective subspace of $R$, then $N \bar{\otimes} T$ is a maximal $\sigma$-weakly closed injective $N \bar{\otimes} \mathbb{C} 1_{R}$-bimodule of $N \bar{\otimes} R$;

(2) if $A$ is a maximal $\sigma$-weakly closed injective unital (but not necessarily selfadjoint) subalgebra of $R$, then $N \bar{\otimes} A$ is a maximal $\sigma$-weakly closed injective unital subalgebra of $N \bar{\otimes} R$.

Proof. (1) We first show that $N \bar{\otimes} T$ is injective. Since $T$ is $\sigma$-weakly closed, it is dual as a Banach space. So it follows from Theorem 1.3 in [1] that there is an injective von Neumann algebra $P$ and a projection $e$ in $P$ such that $T$ is completely isometric and weak* homeomorphic to $e P\left(1_{P}-e\right)$, and hence $N \bar{\otimes} T$ is completely isometric and weak* homeomorphic to $N \bar{\otimes} e P\left(1_{P}-e\right)$. Let $f=1_{N} \otimes e$, and let $M=N \bar{\otimes} P$. Then $1_{M}-f=1_{N} \otimes\left(1_{P}-e\right)$, and so $N \bar{\otimes} e P\left(1_{P}-e\right)=f M\left(1_{M}-f\right)$. Since $N$ and $P$ are injective von Neumann algebras, so is $M$ ([8, Proposition 10.24]). Thus another application of Theorem 1.3 in [1] shows that $N \bar{\otimes} T$ is injective. Now suppose that $S$ is a $\sigma$-weakly closed injective $N \bar{\otimes} \mathbb{C} 1_{R}$-bimodule of $N \bar{\otimes} R$, and that $N \bar{\otimes} T \subset S$. Since $N$ is injective, it has Property $S_{\sigma}$. (It is shown in [6] that semidiscrete von Neumann algebras have Property $S_{\sigma}$, and, as shown in [13], semidiscreteness is equivalent to injectivity.) Hence by Theorem 3, there is a $\sigma$-weakly closed subspace $T_{0}$ of $R$ such that $S=N \bar{\otimes} T_{0}$, and since $\mathbb{C} 1_{N} \bar{\otimes} T \subset S=F\left(N, T_{0}\right), T \subset T_{0}$. Suppose $N \subset B(K)$ for a Hilbert space $K$. Then $S \subset B(K) \bar{\otimes} B(H)=B(K \otimes H)$ is injective. So there is a completely contractive projection $\Psi$ from $B(K \otimes H)$ onto $S$. Let $\phi$ be a normal state of $N$, and let $\Phi$ be the unique normal linear map from $N \bar{\otimes} R$ onto $\mathbb{C} 1_{N} \bar{\otimes} R$ such that $\Phi(a \otimes b)=\phi(a) 1_{N} \otimes b$ for all $a \in N$ and $b \in R$. Then it is easily checked that $\Phi \circ \Psi$ is a completely contractive projection from $B(K \otimes H)$ onto $\mathbb{C} 1_{N} \bar{\otimes} T_{0}$. Hence $\mathbb{C} 1_{N} \bar{\otimes} T_{0}$ is injective, from which it follows easily that $T_{0}$ is injective. By the maximality of $T, T=T_{0}$, and so $S=N \bar{\otimes} T$. Hence $N \bar{\otimes} T$ is a maximal $\sigma$-weakly closed injective $N \bar{\otimes} \mathbb{C} 1_{R}$-bimodule of $N \bar{\otimes} R$.

(2) Let $B$ be a $\sigma$-weakly closed injective unital subalgebra of $N \bar{\otimes} R$ such that $N \bar{\otimes} A \subset B$. Then since $A$ is unital, $N \bar{\otimes} \mathbb{C} 1_{R} \subset B$, and so, since $B$ is an algebra, $B$ is an $N \bar{\otimes} \mathbb{C} 1_{R}$-bimodule. Thus, by the same argument as in (1), $B=N \bar{\otimes} A_{0}$, where $A \subset A_{0}$ and $A_{0}$ is an injective $\sigma$-weakly closed subspace of $R$. Moreover, if $a$ and $b$ are elements of $A_{0}$, then $1_{N} \otimes a$ and $1_{N} \otimes b$ are elements of the algebra $B$; so $1_{N} \otimes a b=\left(1_{N} \otimes a\right)\left(1_{N} \otimes b\right) \in B=F\left(N, A_{0}\right)$, and so $a b \in A_{0}$. Hence $A_{0}$ is also a unital subalgebra of $R$, and so by the maximality of $A, A=A_{0}$, and so $B=N \bar{\otimes} A$. Thus $N \bar{\otimes} A$ is a maximal $\sigma$-weakly closed injective unital subalgebra of $N \bar{\otimes} R$.

We conclude this paper with a result that generalizes the main result in [16] (and Theorem 3.3(1) in [15]). First we need some terminology. If $A$ and $B$ are $\mathrm{C}^{*}$-algebras, we denote by $A \otimes B$ the minimal or spatial tensor product of $A$ and 
B. (If $A \subset B(H)$ and $B \subset B(K)$, then $A \otimes B$ is just the norm closed linear span in $B(H) \bar{\otimes} B(K)$ of $\{a \otimes b: a \in A, b \in B\}$.) Slice maps and Fubini products can be defined as in the von Neumann algebra case, with the $\sigma$-weak topology replaced by the norm topology. (So, for example, if $\phi \in A^{*}$, then the right slice map $R_{\phi}$ is the unique norm bounded linear map from $A \otimes B$ to $B$ satisfying $R_{\phi}(a \otimes b)=\phi(a) b$ for all $a \in A$ and $b \in B$.) In contrast to the case of von Neumann algebras, the Fubuni product depends on $A$ and $B$. (See section 5 of 7 .) Since our first $\mathrm{C}^{*}$-algebra will be fixed, we use the following special notation: if $A$ and $B$ are $\mathrm{C}^{*}$-algebras, and if $T$ is a norm closed subspace of $B$, then

$$
F_{B}(T)=\left\{x \in A \otimes B: R_{\phi}(x) \in T \text { for all } \phi \in A^{*}\right\} .
$$

Note that since every bounded linear functional on $A$ is a linear combination of states, we also have that $F_{B}(T)$ is the norm closed linear span of $\{x \in A \otimes B$ : $R_{\phi}(x) \in T$ for all states $\phi$ of $\left.A\right\}$. A $\mathrm{C}^{*}$-algebra $A$ is said to have Property $S$ for subspaces (see [7]) if for every pair $(T, B)$, where $B$ is a $\mathrm{C}^{*}$-algebra and $T$ is a norm closed subspace of $B$, we have $F_{B}(T)=A \otimes T$. (A $\mathrm{C}^{*}$-algebra is said to have Property $S$ if $F_{B}(C)=A \otimes C$ whenever $C$ is a $\mathrm{C}^{*}$-subalgebra of a $\mathrm{C}^{*}$-algebra $B$. Property $S$ was introduced by Wassermann in [11], and was the inspiration for both Property $S_{\sigma}$ and Property $S$ for subspaces. In [12, Wassermann showed that there are $\mathrm{C}^{*}$-algebras that do not have Property $S$.) It is shown in [7] that a $\mathrm{C}^{*}$-algebra has Property $S$ for subspaces if and only if for any $\mathrm{C}^{*}$-algebra $B$ there is a net $\left\{\Phi_{i}\right\}$ of finite rank completely bounded maps from $A$ to $A$ such that $\left(\Phi_{i} \otimes i d_{B}\right)(x) \rightarrow x$ in the point norm topology of $A \otimes B$. A $\mathrm{C}^{*}$-algebra with this approximation property is said to have the strong operator approximation property, or strong OAP (see p. 185 of [2] or Chapter 11 of [3]). Wassermann showed in [14] that nuclear $\mathrm{C}^{*}$ algebras have Property $S$, and his proof of this result (Proposition 10 in [14]) shows that nuclear $\mathrm{C}^{*}$-algebras have Property $S$ for subspaces (and also shows directly that nuclear $\mathrm{C}^{*}$-algebras have the strong OAP).

The main result (Theorem) in [16] is that if $A, D$ and $C$ are unital $\mathrm{C}^{*}$-algebras, if $A$ is simple and nuclear, and if $A \otimes \mathbb{C}_{D} \subset C \subset A \otimes D$, then $C=A \otimes B$ for some $\mathrm{C}^{*}$-subalgebra $B$ of $D$. This result was obtained independently by Zacharias, using elementary maps (Theorem 3.3(1) of [15]), where it was explicitly observed that the only property of nuclearity that Zsido used was the fact that a nuclear $\mathrm{C}^{*}$-algebra has Property $S$. So the result remains valid if we just assume that $A$ is simple and has Property $S$. Our last theorem follows from a straightforward modification of the proof of [16, Theorem], or the proof of [15, Theorem 3.3(1)]; so the proof is omitted.

Theorem 7. Let $A$ and $B$ be unital $C^{*}$-algebras, with $A$ simple, and suppose $S$ is a norm closed subspace of $A \otimes B$ that is also an $A \otimes \mathbb{C} 1_{B}$-bimodule (under multiplication). Then

(1) there is a norm closed subspace $T$ of $B$ such that $A \otimes T \subset S \subset F_{B}(T)$;

(2) if $A$ has Property $S$ for subspaces, or, equivalently, if $A$ has the strong $O A P$ (in particular, if $A$ is nuclear), then $S=A \otimes T$ for some norm closed subspace $T$ of $B$.

Remark. Theorem 3.3(2) in [15] can also be generalized to the subspace case, with the obvious modifications to the proof. The general result is: Suppose $A$ and $B$ are unital $\mathrm{C}^{*}$-algebras and that $A$ contains a unital abelian $\mathrm{C}^{*}$-subalgebra $D$ that has the pure state extension property (each pure state on $D$ extends uniquely to 
a pure state on $A$ ). Suppose $C$ is a norm closed subspace of $A \otimes B$ that is an $A \otimes \mathbb{C} 1_{B}$-bimodule, and that $B_{\omega}=B_{0}$ for each pure state $\omega$ on $A$ and some norm closed subspace $B_{0}$ of $B$. (See [15] for the definition of $B_{\omega}$. Note that if we just assume $C$ is an $A \otimes \mathbb{C} 1_{B}$-bimodule, then the proofs of (i) and (iii) of Proposition 3.2 in [15] are still valid.) Then $A \otimes B_{0} \subset C \subset F_{B}\left(B_{0}\right)$, and thus $C=A \otimes B_{0}$ if $A$ has Property $\mathrm{S}$ for subspaces.

\section{REFERENCES}

[1] E. G. Effros, N. Ozawa, and Z.-J. Ruan, On injectivity and nuclearity for operator spaces, Duke Math. J. 110 (2001), 489-521. MR 2002k:46151

[2] E. G. Effros and Z.-J. Ruan, On approximation properties for operator spaces, International J. Math. 1 (1990), 163-187. MR 92g:46089

[3] E. Effros and Z.-J. Ruan, Operator Spaces, The Clarendon Press, Oxford University Press, New York, 2000. MR 2002a:46082

[4] L. Ge and R. V. Kadison, On tensor products for von Neumann algebras, Invent. Math. 123 (1996), 453-466. MR 97c:46074

[5] H. Halpern, Module homomorphisms of a von Neumann algebra into its center, Trans. Amer. Math. Soc. 140 (1969), 183-193. MR 39:3321

[6] J. Kraus, The slice map problem for $\sigma$-weakly closed subspaces of von Neumann algebras, Trans. Amer. Math. Soc. 279 (1983), 357-376. MR 85e:46036

[7] J. Kraus, The slice map problem and approximation properties, J. Funct. Anal. 102 (1991), 116-155. MR 92m:47083

[8] S. Strătilă, Modular theory in operator algebras, Abacus Press, Tunbridge Wells, 1981. MR 85g: 46072

[9] S. Strătilă and L. Zsidó, An algebraic reduction theory for $W^{*}$-algebras, I, J. Funct. Anal. 11 (1972), 295-313. MR 49:5875

[10] S. Strătilă and L. Zsidó, The commutation theorem for tensor products over von Neumann algebras, J. Funct. Anal. 165 (1999), 293-346. MR 2000j:46115

[11] S. Wassermann, The slice map problem for $C^{*}$-algebras, Proc. London Math. Soc. (3) 32 (1976), 537-559. MR 53:14152

[12] S. Wassermann, On tensor products of certain group $C^{*}$-algebras, J. Funct. Anal. 23 (1976), 239-254. MR 54:13582

[13] S. Wassermann, Injective $W^{*}$-algebras, Math. Proc. Cambridge Philos. Soc. 82 (1977), 39-47. MR 56:6418

[14] S. Wassermann, A pathology in the ideal space of $L(H) \otimes L(H)$, Indiana Univ. Math. J. 27 (1978), 1011-1020. MR 80d:46113

[15] J. Zacharias, Splitting for subalgebras of tensor products, Proc. Amer. Math. Soc. 129 (2001), 407-413. MR 2001e:46106

[16] L. Zsidó, A criterion for splitting $C^{*}$-algebras in tensor products, Proc. Amer. Math. Soc. 128 (2000), 2001-2006. MR 2000m:46119

Department of Mathematics, State University of New York at Buffalo, Buffalo, NEW YORK 14260-2900

E-mail address: mthjek@acsu.buffalo.edu 